Abstract

\title{
A Life-Course Model of Obesity: Primary and Secondary Interventions to Prevent Childhood Obesity $^{\dagger}$
}

\author{
Lynne Allison Daniels \\ School Exercise and Nutrition Sciences and Institute Health Biomedical Innovation, Queensland University \\ Technology, Brisbane 5049, Australia; 12.daniels@qut.edu.au \\ + Presented at the 2018 Nutrition Society of New Zealand Annual Conference, Auckland, New Zealand, \\ 28-30 November 2018.
}

Published: 10 April 2019

Background: Childhood obesity is highly prevalent globally. It has adverse effects on health and wellbeing and is a key risk factor for adult obesity and associated co-morbidities. The WHO recommends both early-life interventions and weight management services for children already above the healthy weight range. The aim is to describe the rationale, content and outcomes of two interventions that aimed to prevent childhood obesity.

Methods: NOURISH is a universal early feeding intervention that commenced in infancy and promoted protective feeding practices for first time mothers and evaluated in an RCT $(n=697)$. PEACH (Parenting Eating Activity for Child Health) is a targeted secondary prevention (treatment) multi-component lifestyle intervention for parents of overweight primary school-aged children demonstrated to be efficacious in a research setting via RCT. PEACH Queensland (Qld) used prepost evaluation to examine outcomes of delivery of the program at scale ( $n=817$ families) in a 'real world service/practice setting.

Results: NOURISH demonstrated positive outcomes for maternal feeding practices sustained up to 5 years of age, 3 years after intervention completion. For PEACH Qld paired pre-post evaluation data for 388 children showed significant improvements in weight status, parenting self-efficacy and child eating behaviour and activity.

Conclusions: Key challenges included a range of system and service barriers to delivery of sustainable child weight management programs through routine health care and engagement of parents in those programs. 